\title{
Unicellularity of the multiplication operator on Banach spaces of formal power series
}

by

\author{
B. Yousefi (Shiraz)
}

\begin{abstract}
Let $\{\beta(n)\}_{n=0}^{\infty}$ be a sequence of positive numbers and $1 \leq p<\infty$. We consider the space $\ell^{p}(\beta)$ of all power series $f(z)=\sum_{n=0}^{\infty} \widehat{f}(n) z^{n}$ such that $\sum_{n=0}^{\infty}|\widehat{f}(n)|^{p}|\beta(n)|^{p}$ $<\infty$. We give some sufficient conditions for the multiplication operator, $M_{z}$, to be unicellular on the Banach space $\ell^{p}(\beta)$. This generalizes the main results obtained by $\mathrm{Lu}$ Fang [1].
\end{abstract}

Introduction. First, we generalize some definitions from [4].

Let $\{\beta(n)\}$ be a sequence of nonzero complex numbers with $\beta(0)=1$ and $1 \leq p<\infty$. We consider the space of sequences $f=\{\widehat{f}(n)\}_{n=0}^{\infty}$ such that

$$
\|f\|^{p}=\|f\|_{\beta}^{p}=\sum_{n=0}^{\infty}|\widehat{f}(n)|^{p}|\beta(n)|^{p}<\infty .
$$

The notation $f(z)=\sum_{n=0}^{\infty} \widehat{f}(n) z^{n}$ will be used whether or not the series converges for any value of $z$. These are called formal power series. Let $\ell^{p}(\beta)$ denote the space of such formal power series.

For $1<p<\infty, \ell^{p}(\beta) \cong L^{p}(\mu)$ where $\mu$ is the $\sigma$-finite measure defined on the positive integers by $\mu(K)=\sum_{n \in K} \beta(n)^{p}, K \subseteq \mathbb{N} \cup\{0\}$. So $\ell^{p}(\beta)$ is a reflexive Banach space $([3])$ and $\left(\ell^{p}(\beta)\right)^{*}=\ell^{q}\left(\beta^{p / q}\right)$ where $\beta^{p / q}=$ $\left\{\beta(n)^{p / q}\right\}_{n}([6])$.

Let $\widehat{f}_{k}(n)=\delta_{n k}$. So $f_{k}(z)=z^{k}$ and then $\left\{f_{k}\right\}_{k}$ is a basis such that $\left\|f_{k}\right\|=|\beta(k)|$. Now consider $M_{z}$, the operator of multiplication by $z$ on $\ell^{p}(\beta)$ :

$$
\left(M_{z} f\right)(z)=\sum_{n=0}^{\infty} \widehat{f}(n) z^{n+1} .
$$

2000 Mathematics Subject Classification: Primary 47B37; Secondary 47A25.

Key words and phrases: invariant subspace lattice, Banach space of formal power series associated with a sequence $\beta$, cyclic vector, unicellular operator.

Research partially supported by a grant from Shiraz University Research Council. 
In other words

$$
\left(M_{z} f\right)^{\wedge}(n)= \begin{cases}\widehat{f}(n-1), & n \geq 1, \\ 0, & n=0 .\end{cases}
$$

Clearly $M_{z}$ shifts the basis $\left\{f_{k}\right\}_{k}$. The operator $M_{z}$ is bounded if and only if $\{\beta(k+1) / \beta(k)\}_{k}$ is bounded and in this case

$$
\left\|M_{z}^{n}\right\|=\sup _{k}\left|\frac{\beta(n+k)}{\beta(k)}\right|, \quad n=0,1,2, \ldots
$$

Consider the multiplication of formal power series, $f g=h$, given by

$$
\left(\sum_{n=0}^{\infty} \widehat{f}(n) z^{n}\right) \cdot\left(\sum_{n=0}^{\infty} \widehat{g}(n) z^{n}\right)=\sum_{n=0}^{\infty} \widehat{h}(n) z^{n}
$$

where

$$
\widehat{h}(n)=\sum_{k=0}^{n} \widehat{f}(k) \widehat{g}(n-k), \quad n=0,1,2, \ldots
$$

If $1 / p+1 / q=1$ and

$$
\sup _{n} \sum_{i=1}^{n}\left|\frac{\beta(n)}{\beta(i) \beta(n-i)}\right|^{q}<\infty
$$

then clearly by the Hölder inequality one can see that $\ell^{p}(\beta)$ is a Banach algebra $([2])$.

If $f \in \ell^{p}(\beta)$ and $P(z)$ is a polynomial, then to the vector $P\left(M_{z}\right) f$ there corresponds the formal power series $P(z) f(z)$.

Let $X$ be a Banach space. We denote by $B(X)$ the set of bounded linear operators on $X$. Let $A \in B(X)$ and $x \in X$. We say that $x$ is a cyclic vector of $A$ if

$$
X=\operatorname{span}\left\{A^{n} x: n=0,1,2, \ldots\right\} .
$$

Here $\operatorname{span}\{\cdot\}$ is the closed linear span of the set $\{\cdot\}$. A polynomial $p(z)=$ $\left(z-\lambda_{1}\right) \ldots\left(z-\lambda_{k}\right)$ is a cyclic vector of $M_{z}$ in $\ell^{p}(\beta)$ iff $\left\{\lambda_{i}^{n} / \beta(n)\right\}_{n} \notin \ell^{q}$ for $i=1, \ldots, k$, where $1 / p+1 / q=1([6])$.

Also an operator $A$ in $B(X)$ is called unicellular on $X$ if the set of its invariant subspaces, $\operatorname{Lat}(A)$, is linearly ordered by inclusion.

In the main theorem of this paper we give some sufficient conditions for the multiplication operator, $M_{z}$, on $\ell^{p}(\beta)$ to be unicellular and then we obtain the main results of [1]. Throughout this paper we assume that $M_{z} \in B\left(\ell^{p}(\beta)\right)$.

Unicellularity of $M_{z}$. The following theorem is the main result of this paper.

Theorem. Let $1 \leq p<\infty$. The operator $M_{z}$ is unicellular on $\ell^{p}(\beta)$ if $\beta(n)$ is of the form $\beta(n)=\alpha(n) \gamma(n)$ where $\{\alpha(n)\}$ and $\{\gamma(n)\}$ satisfy: 
(i) There exists a positive number $M$ such that

$$
\sup \left\{\left|\frac{\gamma(n+i)}{\gamma(n) \gamma(i)}\right|: i, n=0,1,2, \ldots\right\} \leq M .
$$

(ii) There exists a positive integer $m_{0}$ such that

$$
L_{m_{0}}=\sup \left\{\left|\frac{\alpha(n+i) \alpha\left(m_{0}\right)}{\alpha\left(n+m_{0}\right) \alpha(i)}\right|: n>0, i \geq m_{0}\right\}<\infty
$$

and

$$
\left\{\frac{\alpha\left(n+m_{0}\right)}{\alpha(n)}\right\}_{n} \in \ell^{q}
$$

where $1 / p+1 / q=1$.

Proof. Let $\left\{f_{m}\right\}_{m}$ be the basis for $\ell^{p}(\beta)$ as defined in the introduction. Put $\ell_{\infty}^{p}(\beta)=\{0\}, \ell_{0}^{p}(\beta)=\ell^{p}(\beta)$ and

$$
\ell_{n}^{p}(\beta)=\left\{\sum_{m \geq n} c_{m} f_{m} \in \ell^{p}(\beta)\right\} \quad(n \geq 1) .
$$

In order to show that $M_{z}$ is unicellular it suffices to show that the lattice of invariant subspaces of $M_{z}$, Lat $\left(M_{z}\right)$, is a subset of $\left\{\ell_{n}^{p}(\beta): 0 \leq n \leq \infty\right\}$. So let $\mathcal{K}$ be a nontrivial element of $\operatorname{Lat}\left(M_{z}\right)$. Then there exists a positive integer $n$ such that $\mathcal{K} \subseteq \ell_{n}^{p}(\beta)$ and $\mathcal{K} \nsubseteq \ell_{n+1}^{p}(\beta)$. Thus we may choose $f=\sum_{m=n}^{\infty} x_{m} f_{m}$ in $\mathcal{K}\left(x_{m}=\widehat{f}(m)\right)$ with $x_{n} \neq 0$. Note that $\left\{f_{n+k}\right\}_{k=0}^{\infty}$ is a basis for $\ell_{n}^{p}(\beta)$. We claim that $f$ is a cyclic vector for $\left.M_{z}\right|_{\ell_{n}^{p}(\beta)}$. If so, then since $M_{z} \mathcal{K} \subset \mathcal{K}$, we have $M_{z}^{i} f \in \mathcal{K}$ for $i \in \mathbb{N}$. Also since

$$
\ell_{n}^{p}(\beta)=\operatorname{span}\left\{\left(\left.M_{z}^{i}\right|_{\ell_{n}^{p}(\beta)}\right) f: i=0,1,2, \ldots\right\},
$$

we have $\ell_{n}^{p}(\beta) \subseteq \mathcal{K}$ and so $\ell_{n}^{p}(\beta)=\mathcal{K}$. Now to prove our claim it is sufficient to show that if

$$
f=\sum_{m=0}^{\infty} \widehat{f}(m) f_{m} \in \ell^{p}(\beta)
$$

is such that $\widehat{f}(0) \neq 0$, then $f$ is a cyclic vector for $M_{z}$. Without loss of generality, assume that $\widehat{f}(0)=1$. Note that $M_{z} f_{k}=f_{k+1}$. For the formal power series

$$
f(z)=\sum_{m=0}^{\infty} \widehat{f}(m) z^{m}
$$

we choose the formal power series

$$
g(z)=\sum_{m=0}^{\infty} \widehat{g}(m) z^{m}
$$


such that $g(z) f(z)=1$. Indeed $\widehat{g}(0)=1$ and for $k \geq 1$,

$$
\widehat{g}(k)=\sum_{i=1}^{k} \sum_{\substack{m_{1}+\ldots+m_{i}=k \\ m_{j} \geq 1}}(-1)^{i} \widehat{f}\left(m_{1}\right) \ldots \widehat{f}\left(m_{i}\right)
$$

(see [5]). In order to show that $f$ is a cyclic vector of $M_{z}$, we show that

$$
\operatorname{span}\left\{\sum_{k=0}^{m_{0}} \widehat{g}(k) M_{z}^{k+n} f: n=0,1,2, \ldots\right\}=\ell^{p}(\beta)
$$

( $m_{0}$ is the positive integer in condition (ii) of the theorem). Put

$$
y_{m_{0}, n}=\sum_{k=0}^{m_{0}} \widehat{g}(k) M_{z}^{k+n} f, \quad n=0,1,2, \ldots
$$

If there exists a positive integer $n_{0}$ such that

$$
\operatorname{span}\left\{y_{m_{0}, n}: n \geq n_{0}\right\}=\ell_{n_{0}}^{p}(\beta)
$$

then clearly one can see that

$$
\operatorname{span}\left\{y_{m_{0}, n}: n \geq n_{0}-1\right\}=\ell_{n_{0}-1}^{p}(\beta) .
$$

By continuing this process, we conclude that (1) holds. Now since $g(z) f(z)$ $=1$, we have

$$
\left(\sum_{k=0}^{m_{0}} \widehat{g}(k) z^{k}+\sum_{k>m_{0}} \widehat{g}(k) z^{k}\right) f(z)=1
$$

and so

$$
\sum_{k=0}^{m_{0}}\left(\widehat{g}(k) M_{z}^{k}\right) f\left(M_{z}\right) f_{0}+\sum_{k>m_{0}}\left(\widehat{g}(k) M_{z}^{k}\right) f\left(M_{z}\right) f_{0}=f_{0} .
$$

Now since for each $n \geq 0, M_{z}^{n} f_{0}=f_{n}$, by taking the image under $M_{z}^{n}$ of both sides of the above equation, we have

$$
\sum_{k=0}^{m_{0}}\left(\widehat{g}(k) M_{z}^{k+n}\right) f\left(M_{z}\right) f_{0}-f_{n}=-\sum_{k>m_{0}}\left(\widehat{g}(k) M_{z}^{k+n}\right) f\left(M_{z}\right) f_{0} .
$$

Note that $f\left(M_{z}\right) f_{0}=f$ and $f=\sum_{m=0}^{\infty} \widehat{f}(m) f_{m}$. So

$$
y_{m_{0}, n}-f_{n}=\sum_{k>m_{0}} \sum_{m=0}^{\infty} \widehat{g}(k) \widehat{f}(m) M_{z}^{k+n} f_{m} .
$$

Therefore

$$
y_{m_{0}, n}-f_{n} \in \ell_{m_{0}+n+1}^{p}(\beta) .
$$


Now we show that there exists a positive integer $n_{0}$ such that (2) holds. For $i \geq 1$, define the projections $P_{i}: \ell^{p}(\beta) \rightarrow \ell_{i}^{p}(\beta)$ by

$$
P_{i}\left(\sum_{n=0}^{\infty} \widehat{f}(n) z^{n}\right)=\sum_{n=i}^{\infty} \widehat{f}(n) z^{n} .
$$

Note that $\left\|\left.M_{z}^{n}\right|_{\ell_{i}^{p}(\beta)}\right\|=\sup _{m}|\beta(i+n+m) / \beta(i+m)|$ and for $i \geq k, P_{i} M_{z}^{k} f$ $=M_{z}^{k} P_{i-k} f$ for all $f \in \ell^{p}(\beta)([5])$. Thus

$$
\begin{aligned}
\frac{1}{|\beta(n)|}\left\|y_{m_{0}, n}-f_{n}\right\|_{p} & =\frac{1}{|\beta(n)|}\left\|P_{m_{0}+n+1}\left(y_{m_{0}, n}-f_{n}\right)\right\|_{p} \\
& =\frac{1}{|\beta(n)|}\left\|P_{m_{0}+n+1}\left(y_{m_{0}, n}\right)\right\|_{p} \\
& \leq \frac{1}{|\beta(n)|} \sum_{k=0}^{m_{0}}|\widehat{g}(k)| \cdot\left\|P_{m_{0}+n+1} M_{z}^{k+n} f\right\|_{p} \\
& =\frac{1}{|\beta(n)|} \sum_{k=0}^{m_{0}}|\widehat{g}(k)| \cdot\left\|M_{z}^{k+n} P_{m_{0}-k+1} f\right\|_{p} \\
& \leq \frac{\|f\|_{p}}{|\beta(n)|} \sum_{k=0}^{m_{0}}|\widehat{g}(k)| \cdot\left\|\left.M_{z}^{k+n}\right|_{\ell_{m_{0}-k+1}^{p}(\beta)}\right\| \\
& =\|f\|_{p} \sum_{k=0}^{m_{0}}|\widehat{g}(k)| \sup _{i}\left|\frac{\beta\left(m_{0}+n+i+1\right)}{\beta(n) \beta\left(m_{0}+i+1-k\right)}\right| .
\end{aligned}
$$

Since $\beta(n)=\alpha(n) \gamma(n)$, we have

$$
\begin{aligned}
& \sup _{i}\left|\frac{\beta\left(m_{0}+n+i+1\right)}{\beta(n) \beta\left(m_{0}+i+1-k\right)}\right| \\
& \quad=\sup _{i}\left|\frac{\alpha\left(m_{0}+n+i+1\right)}{\alpha(n) \alpha\left(m_{0}+i+1-k\right)}\right|\left|\frac{\gamma\left(m_{0}+n+i+1\right)}{\gamma(n) \gamma\left(m_{0}+i+1-k\right)}\right| .
\end{aligned}
$$

But by condition (ii) of the theorem,

$$
\begin{aligned}
\sup _{i}\left|\frac{\alpha\left(m_{0}+n+i+1\right)}{\alpha(n) \alpha\left(m_{0}+i+1-k\right)}\right| \\
\quad=\sup _{i}\left|\frac{\alpha\left(m_{0}+n+i+1\right) \alpha\left(m_{0}\right)}{\alpha\left(m_{0}+n\right) \alpha\left(m_{0}+i+1\right)}\right|\left|\frac{\alpha\left(m_{0}+i+1\right) \alpha\left(m_{0}+n\right)}{\alpha(n) \alpha\left(m_{0}\right) \alpha\left(m_{0}+i+1-k\right)}\right| \\
\quad \leq L_{m_{0}}\left|\frac{\alpha\left(m_{0}+n\right)}{\alpha(n) \alpha\left(m_{0}\right)}\right| \sup _{i}\left|\frac{\alpha\left(m_{0}+i+1\right)}{\alpha\left(m_{0}+i+1-k\right)}\right|,
\end{aligned}
$$

and by (i), 


$$
\begin{gathered}
\sup _{i}\left|\frac{\gamma\left(m_{0}+n+i+1\right)}{\gamma(n) \gamma\left(m_{0}+i+1-k\right)}\right| \\
\quad=\sup _{i}\left|\frac{\gamma\left(m_{0}+n+i+1\right)}{\gamma(n) \gamma\left(m_{0}+i+1\right)}\right|\left|\frac{\gamma\left(m_{0}+i+1\right)}{\gamma\left(m_{0}+i+1-k\right)}\right| \\
\leq M \sup _{i}\left|\frac{\gamma\left(m_{0}+i+1\right)}{\gamma\left(m_{0}+i+1-k\right)}\right| .
\end{gathered}
$$

So

$$
\begin{aligned}
& \sup _{i}\left|\frac{\beta\left(m_{0}+n+i+1\right)}{\beta(n) \beta\left(m_{0}+i+1-k\right)}\right| \\
& \leq M L_{m_{0}}\left|\frac{\alpha\left(m_{0}+n\right)}{\alpha(n) \alpha\left(m_{0}\right)}\right| \sup _{i}\left|\frac{\beta\left(m_{0}+i+1\right)}{\beta\left(m_{0}+i+1-k\right)}\right|
\end{aligned}
$$

and therefore

$$
\begin{aligned}
& \frac{1}{|\beta(n)|}\left\|y_{m_{0}, n}-f_{n}\right\|_{p} \\
& \quad \leq M L_{m_{0}}\left|\frac{\alpha\left(m_{0}+n\right)}{\alpha(n) \alpha\left(m_{0}\right)}\right|\|f\|_{p} \sum_{k=0}^{m_{0}}|\widehat{g}(k)| \sup _{i}\left|\frac{\beta\left(m_{0}+i+1\right)}{\beta\left(m_{0}+i+1-k\right)}\right| .
\end{aligned}
$$

Since

$$
\left\|M_{z}^{k}\left|\ell_{m_{0}+1-k}^{p}(\beta) \|=\sup _{i}\right| \frac{\beta\left(m_{0}+1+i\right)}{\beta\left(m_{0}+1-k+i\right)} \mid<\infty\right.
$$

for $k=0,1,2, \ldots, m_{0}$, there exists a positive number $M^{\prime}$ such that

$$
\sum_{k=0}^{m_{0}}|\widehat{g}(k)| \sup _{i}\left|\frac{\beta\left(m_{0}+1+i\right)}{\beta\left(m_{0}+1-k+i\right)}\right| \leq M^{\prime}
$$

So we have

$$
\frac{1}{|\beta(n)|}\left\|y_{m_{0}, n}-f_{n}\right\|_{p} \leq c_{n}
$$

where

$$
c_{n}=M M^{\prime}\|f\|_{p} \frac{L_{m_{0}}}{\left|\alpha\left(m_{0}\right)\right|}\left|\frac{\alpha\left(m_{0}+n\right)}{\alpha(n)}\right|, \quad n=1,2, \ldots
$$

Since $\left\{c_{n}\right\} \in \ell^{q}$, there exists a positive integer $n_{0}>m_{0}$ such that

$$
\lambda=\sum_{n>n_{0}} c_{n}^{q}<1
$$

Therefore for any finite linear combinations

$$
\phi=\sum d_{k} y_{m_{0}, n_{0}+k} / \beta\left(n_{0}+k\right), \quad \psi=\sum d_{k} f_{n_{0}+k} / \beta\left(n_{0}+k\right),
$$


by the Hölder inequality we have

$$
\begin{aligned}
\|\phi-\psi\|_{p} & \leq \sum_{k}\left|d_{k}\right| \cdot\left\|y_{m_{0}, n_{0}+k}-f_{n_{0}+k}\right\|_{p} /\left|\beta\left(n_{0}+k\right)\right| \\
& \leq\left(\sum_{k}\left|d_{k}\right|^{p}\right)^{1 / p}\left(\sum_{n=n_{0}}^{\infty}\left\|y_{m_{0}, n}-f_{n}\right\|_{p}^{q}|\beta(n)|^{-q}\right)^{1 / q} \\
& =\|\psi\|_{p}\left(\sum_{n \geq n_{0}} c_{n}^{q}\right)^{1 / q} .
\end{aligned}
$$

Thus

$$
\|\phi-\psi\|_{p} \leq \lambda^{1 / q}\|\psi\|_{p}
$$

Since $0 \leq \lambda^{1 / q}<1,\left\{y_{m_{0}, n}\right\}_{n=n_{0}}^{\infty}$ is in $\ell_{n_{0}}^{p}(\beta)$ and $\left\{f_{n}\right\}_{n \geq n_{0}}$ is a basis for $\ell_{n_{0}}^{p}(\beta)$, it follows immediately from Lemma 2.1 of [1] (which is true for Banach spaces) that $\left\{y_{m_{0}, n}\right\}_{n \geq n_{0}}$ is a complete set, i.e., spanning $\ell_{n_{0}}^{p}(\beta)$. So (2) holds and this completes the proof.

From the proof of the theorem, we obtain the following corollary.

COROLlary. Under the hypothesis of the theorem, if $x=\sum_{m=0}^{\infty} x_{m} f_{m}$ belongs to $\ell^{p}(\beta)$ and $x_{0} \neq 0$, then $x$ is a cyclic vector of $M_{z}$.

Now as a consequence of the above theorem, in the following example we prove the main result of [1] which gives sufficient conditions for a Lambert weighted shift operator to be unicellular.

ExAMPLE. Let $H$ be a separable Hilbert space with orthonormal basis $\left\{e_{n}\right\}_{n=0}^{\infty}$. A unilateral weighted shift operator $S$ in $B(H)\left(S e_{n}=w_{n} e_{n+1}\right)$ is called a Lambert weighted shift operator if the weights $\left\{w_{n}\right\}$ are given by

$$
w_{n}=a_{n} \frac{\left\|A^{n+1} f\right\|}{\left\|A^{n} f\right\|}, \quad n=0,1,2, \ldots,
$$

where $A$ is a given injective operator in $B(H), f$ is a nonzero vector in $H$ and $\left\{a_{n}\right\}_{n=0}^{\infty}$ is a bounded sequence of positive numbers. $S$ is unitarily equivalent to the multiplication operator $M_{z}$ on the space $\ell^{2}(\beta)$ where the sequence $\beta=\{\beta(n)\}_{n=0}^{\infty}$ satisfies $\beta(0)=1$ and

$$
\beta(n)=w_{0} w_{1} \ldots w_{n-1} \quad(n \geq 1) .
$$

The equivalence of these operators is realized by means of the isomorphism $U$ of $\ell^{2}(\beta)$ onto $H$ defined by the formula $(U f)_{n}=\widehat{f}(n) \beta(n)([4])$. Now for each nonnegative integer $n$ put

$$
\alpha(n)=a_{0} \ldots a_{n-1}, \quad \gamma(n)=\left\|A^{n} f\right\| /\|f\| .
$$

If $\{\alpha(n)\}$ and $\{\gamma(n)\}$ satisfy the hypothesis of the theorem, then the Lambert weighted shift operator is unicellular. 
Proposition. Suppose

$$
\sum_{i, n}\left|\frac{\beta(i+n)}{\beta(i) \beta(n)}\right|^{q}<\infty \quad \text { where } 1 / p+1 / q=1 .
$$

Then $M_{z}$ is uicellular on $\ell^{p}(\beta)$.

Proof. Let $\ell_{n}^{p}(\beta)$ be defined as in the proof of the previous theorem. As in that proof, it is sufficient to show that if $f=\sum_{n \geq 0} \widehat{f}(n) f_{n} \in \ell^{p}(\beta)$ is such that $\widehat{f}(0) \neq 0$, then $f$ is a cyclic vector for $M_{z}$. Without loss of generality, assume that $\widehat{f}(0)=1$. Put $y_{n}=M_{z}^{n} f$ for $n=0,1,2, \ldots$ As before we can see that if

$$
\exists n_{0} \in \mathbb{N}, \quad \operatorname{span}\left\{y_{n}: n \geq n_{0}\right\}=\ell_{n_{0}}^{p}(\beta)
$$

then

$$
\operatorname{span}\left\{y_{n}: n \geq n_{0}-1\right\}=\ell_{n_{0}-1}^{p}(\beta) .
$$

By continuing this process we can conclude that $f$ is a cyclic vector. Note that

$$
y_{n}=f_{n}+\sum_{i \geq 1} \widehat{f}(i) f_{i+n}, \quad n \geq 0 .
$$

Now we have

$$
\begin{aligned}
\frac{1}{|\beta(n)|}\left\|y_{n}-f_{n}\right\|_{p} & =\frac{1}{\beta(n) \mid}\left\|\sum_{i \geq 1} \widehat{f}(i) f_{i+n}\right\|_{p} \\
& \leq \sum_{i \geq 1}|\widehat{f}(i)| \cdot|\beta(i)|\left|\frac{\beta(i+n)}{\beta(i) \beta(n)}\right| \\
& \leq\left(\sum_{i \geq 1}|\widehat{f}(i)|^{p}|\beta(i)|^{p}\right)^{1 / p}\left(\sum_{i \geq 1}\left|\frac{\beta(i+n)}{\beta(i) \beta(n)}\right|^{q}\right)^{1 / q} \\
& \leq\|f\|_{p}\left(\sum_{i \geq 1}\left|\frac{\beta(i+n)}{\beta(i) \beta(n)}\right|^{q}\right)^{1 / q} .
\end{aligned}
$$

Put

$$
c_{n}=\|f\|_{p}^{q} \sum_{i \geq 1}\left|\frac{\beta(i+n)}{\beta(i) \beta(n)}\right|^{q}, \quad n=0,1,2, \ldots
$$

Thus $\sum_{n \geq 0} c_{n}<\infty$ and so there exists a positive integer $n_{0}$ such that $\lambda=\sum_{n \geq n_{0}} c_{n}<1$. Therefore for any finite linear combinations

$$
\phi=\sum_{k} c_{k} y_{n_{0}+k}, \quad \chi=\sum_{k} c_{k} f_{n_{0}+k}
$$


we have

$$
\begin{aligned}
\|\phi-\chi\|_{p} & =\left\|\sum_{k} c_{k}\left(y_{n_{0}+k}-f_{n_{0}+k}\right)\right\|_{p} \\
& \leq\|\chi\|_{p}\left(\sum_{n \geq n_{0}} \frac{\left\|y_{n}-f_{n}\right\|_{p}^{q}}{|\beta(n)|^{q}}\right)^{1 / q}=\lambda^{1 / q}\|\chi\|_{p} .
\end{aligned}
$$

Since $\left\{f_{n_{0}+k}\right\}_{k \geq 0}$ is a basis for $K_{n_{0}}$, and $0 \leq \lambda^{1 / q}<1$, it follows that (1) holds. This completes the proof.

\section{References}

[1] L. Fang, Unicellularity of Lambert's weighted shifts, Northeastern Math. J. 7 (1991), $35-40$.

[2] N. K. Nikol'skiū, Selected problems of weighted approximation and spectral analysis, Trudy Mat. Inst. Steklov. 120 (1974) (in Russian).

[3] K. Seddighi, K. Hedayatiyan and B. Yousefi, Operators acting on certain Banach spaces of analytic functions, Internat. J. Math. Math. Sci. 18 (1995), 107-110.

[4] A. L. Shields, Weighted shift operators and analytic function theory, in: Math. Surveys 13, Amer. Math. Soc., Providence, 1974, 49-128.

[5] D. V. Yakubovich, Conditions for unicellularity of weighted shift operators, Dokl. Akad. Nauk SSSR 278 (1984), 821-824 (in Russian).

[6] B. Yousefi, On the space $\ell^{p}(\beta)$, Rend. Circ. Mat. Palermo 49 (2000), 115-120.

College of Sciences

Shiraz University

Shiraz 71454, Iran

E-mail: yousefi@math5.susc.ac.ir

Received January 8, 1999

Revised version November 20, 2000 